Original Article

\title{
Traditional Korean medicine theory based-therapeutic potential of Gung-Gwi-Tang on postpartum obesity: psychosocial aspects of postpartum obesity
}

\author{
Jeong-Hwa Kim ${ }^{1}$, Phil-Dong Moon ${ }^{2, *}$ \\ ${ }^{1}$ Graduate School of Social Welfare, Ewha Womans University 11-1 Daehyun-Dong, Seodaemun-Gu, Seoul, Republic of Korea; \\ ${ }^{2}$ Department of Pharmacology, College of Oriental Medicine, Institute of Oriental Medicine, Kyung Hee University, 1 Hoegi-dong, \\ Dongdaemun-gu, Seoul, Republic of Korea
}

\begin{abstract}
Obesity is psychological and socioeconomic problems as well as health problems related to physical disease and disorder. The obesity epidemic, including a marked increase in the prevalence of obesity among pregnant women, represents a critical public health problem throughout the world. Gung-Gui-Tang (GGT), a prescription of traditional Korean medicine, has been used to treat dizziness due to loss of blood as well as static blood after childbirth. However, the therapeutic potential of GGT on postpartum obesity has not been fully elucidated in an experimental model. In our research, GGT inhibited the increases of body weight and adipose tissues in postpartum mice fed a high-fat diet. GGT also inhibited the elevations of plasma lipid profiles such as triglyceride, low-density lipoprotein cholesterol, total cholesterol, and glutamate pyruvate transaminase. Overall, these results provide evidence that GGT can help to inhibit postpartum obesity and open new perspective to recover the shape of mother into the moment of conception.
\end{abstract}

Keywords Gung-Gui-Tang, postpartum obesity, body weight, high-fat diet, plasma lipid profiles

\section{INTRODUCTION}

Extensive research supports that obesity during the postpartum period presents the psychosocial consequences of being fat (Pedersen et al., 2011). Postpartum is the period of transition to motherhood (Davis et al, 2012). Most women in postpartum stage experience certain amount of distress due to changes of lifestyle including breastfeeding, managing nutrition and sleep patterns (Pedersen, et al., 2011). Researchers demonstrate a strong connection between obesity and depression among postpartum women (Schmitt et al., 2007). Overweight and obese women in the postpartum period have a greater rate of depression than normal weight women (Carter et al., 2000). Obesity is also associated with Postpartum depression(PPD) which is mood disorder involving symptoms of fatigue, fears, anxiety, thoughts of compulsion, and mood swings (Sundaram et al., 2012). Postpartum depression causes neglect of the infant, child care stress, poor marital satisfaction, and low self-esteem (Reck et al., 2004). Regarding socioeconomic factors, researches are consistently reported that the level of socioeconomic status is inversely correlated with obesity in developed countries (Gustafsson et al., 2012). Low income women with less than a high school education have twice the obesity rates as women of higher socioeconomic status (Singh et al., 2010).

The prevalence of obesity has increased over the past decades (Stienstra et al., 2007). A certain amount of body fat is necessary for storing energy, heat insulation, shock absorption,

\footnotetext{
*Correspondence: Phil-Dong Moon

E-mail: pdmoon@khu.ac.kr

Received May 21, 2011; Accepted August 27, 2012; Published August 31, 2012

doi: http://dx.doi.org/10.5667/tang.2011.0003

(C)2012 by Association of Humanitas Medicine

TANG / www.e-tang.org
}

and other functions. The normal amount of body fat is between $25-30 \%$ in women and $18-23 \%$ in men. Women with over $30 \%$ body fat and men with over $25 \%$ body fat are considered obese (Mokdad et al., 2001). Obesity increases the risk of various diseases, particularly heart disease, type 2 diabetes, breathing difficulties during sleep, certain types of cancer, and osteoarthritis (Rasouli and Kern, 2008).

In traditional Korean medicine (TKM), obesity was described with the characters 'obese (肥)' and 'obese person (肥人)' in ancient medical book (Hong, 1985). The etiology according to the figure of the person explains the cause of a disease with simple sentences like 'the obese person has much dampness (肥人濕多)' and 'the lean person has much fire (瘦 人火多) (Huh, 1999). In general, in TKM, the causes of obesity are seen as the overeating of oily foods (過食 膏粱厚味), qi deficiency (氣虛, deficiency of qi that leads to decreased visceral functions and lowered body resistance), phlegm-dampness (痰濕, a combination of phlegm and internal dampness as an entity to cause disease), and static blood (瘀血, a pathological product of blood stagnation, including extravasated blood and the blood circulating sluggishly or blood congested in a viscus, all of which may turn into a pathogenic factor) (Kim and Choi, 1985).

Most women have a tendency to be fat after childbirth. This postpartum obesity is mainly induced by the dual deficiency of spleen-kidney (脾腎兩虛) and qi deficiency with blood stasis (氣虛血瘀) (Kim et al., 2007). We focused on the treatment of blood stasis to treat postpartum obesity because a number of clinical doctors of TKM have used Gung-Gwi-Tang (GGT) to help induce the removal of lochia (惡露, vaginal discharge in the puerperium). GGT, a prescription of TKM, has been used to treat dizziness due to loss of blood (血軍) as well as static 
blood after childbirth (産後疮血) (Huh, 1999). Indeed, Cnidii Rhizoma (Cnidium officinale Makino; Umbelliferae), a component of GGT, increased the regional cerebral blood flow in rats (Choi, 2004). Because GGT also has following effects such as activating blood (活血) and the removal of static blood (去瘀血) (Huh, 1999), we thus hypothesize that GGT would be effective for the treatment of postpartum obesity.

In this study, we used a high-fat (HF) diet-induced obese mouse model. To create the same conditions as for a mother after childbirth, we used postpartum mice. Thus, we investigated the effect of GGT in a HF diet-induced obese model using postpartum mice.

\section{MATERIALS and METHODS}

\section{Preparation of GGT}

GGT (voucher No 201053), which is a mixture of 2 traditional herbal drugs, was obtained from Noa Oriental Pharmacy (Seoul, Republic of Korea). A prescription of GGT consists of Cnidii Rhizoma (Cnidium officinale Makino; Umbelliferae) and Angelicae Gigantis Radix (Angelica gigas Nakai; Umbelliferae) in the ratio of 1:1. An extract of GGT was prepared by decocting the two dried herbal medicine with distilled water (DW). The extract was decocted for approximately $2 \mathrm{~h}$, filtered, lyophilized, and kept at $4{ }^{\circ} \mathrm{C}$. The yield of GGT was about $5 \%$ $(\mathrm{w} / \mathrm{w})$. Dilutions were made in DW and filtered through a $0.22-\mu \mathrm{m}$ syringe filter.

\section{Experimental design}

Female postpartum C57BL/6N mice were purchased from Daehan Biolink (Daejeon, Republic of Korea). The animals were all maintained in conditions in accordance with those recommended in the NIH Guide for the Care and Use of
Laboratory Animals. The animals were maintained under a $12 \mathrm{~h}$ light/dark cycle at a constant temperature of $22 \pm 3^{\circ} \mathrm{C}$. Three groups of mice were fed with 1) a control (CON) diet (Research Diets, D12450B, 10\% $\mathrm{kcal} \%$ fat) + oral administration of DW (twice a week) or 2) a 'very high-fat' (HF) diet (Research Diets, D12492, 60\% kcal\% fat) + oral administration of DW (twice a week) or 3) a HF diet + oral administration of GGT (1 g/kg, twice a week). All animals were given free access to food and tap water for 8 weeks. Body weight was recorded every week. At the end of this period, the animals were fasted overnight. The next day, they were anesthetized with zoletile and rompun (1:2) and then blood samples were collected by cardiac puncture. The epididymal fat pads were immediately weighed.

\section{Plasma analysis}

Plasma was separated immediately after the collection of blood samples by centrifugation at $10,000 \times \mathrm{g}$ for $10 \mathrm{~min}$. Plasma concentrations of total cholesterol were determined using automated enzymatic methods (Pitt et al., 1995), and low-density lipoprotein (LDL) cholesterol was calculated using the Friedewald formula (Friedewald et al., 1972). Plasma concentrations of triglyceride were measured enzymatically using a triglyceride assay kit (Asan Co. Seoul, Republic of Korea). Glutamate pyruvate transaminase (GPT) activity was determined with an ALT/GPT kit (Sigma-Aldrich, St Louis, MO, USA).

\section{Statistical analysis}

Results are expressed as the mean \pm standard error of the mean (S.E.M.). The statistical evaluation of the results was performed by an independent $t$-test and all statistical analyses were performed using SPSS v11.0 statistical analysis software. The results are significant with a value of $p<0.05$.
A

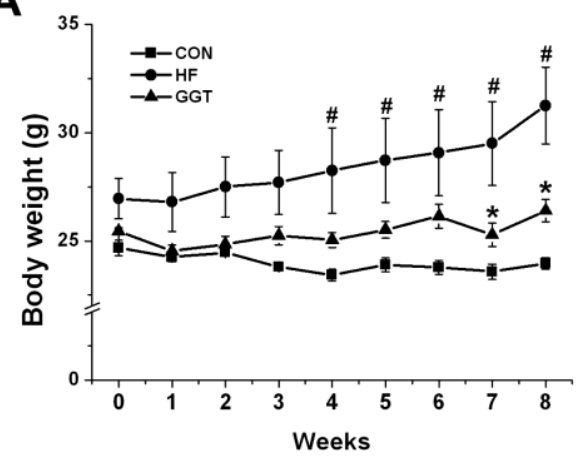

B

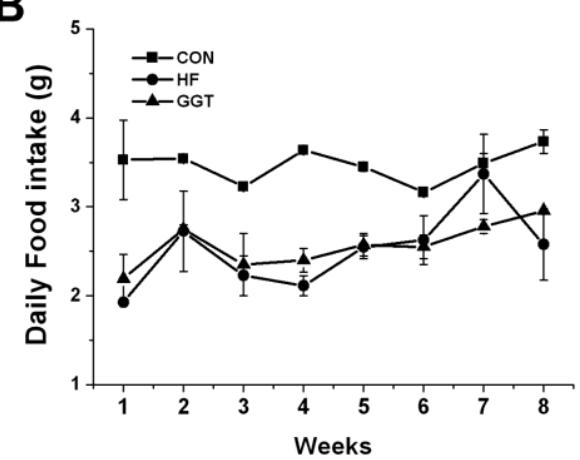

C

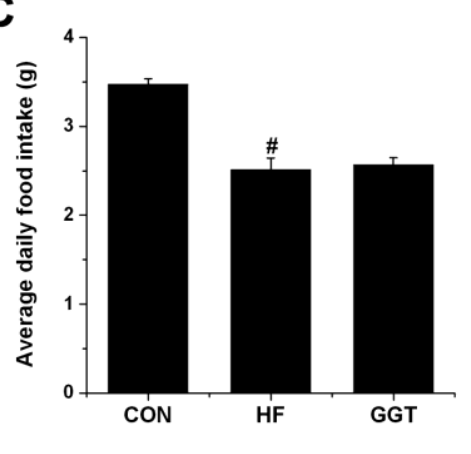

D

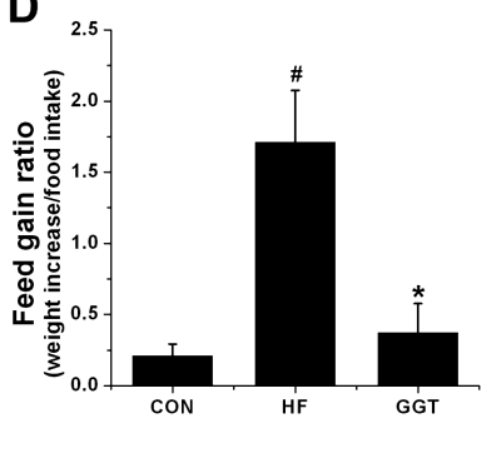

E

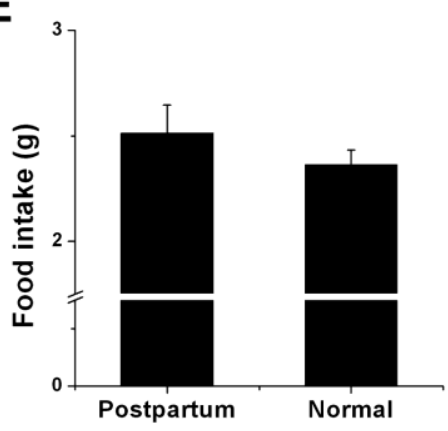

F

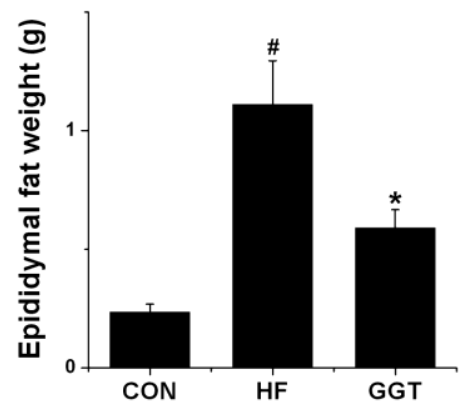

Fig. 1. Effects of GGT on HF diet-induced increase of weight. (A) Effect of GGT on the weight of the mice for 8 weeks. (B) Amount of food intake every week. (C) Average daily food intake. (D) Feed gain ratio. The values are weight increase divided by food intake. (E) Comparison of food intake between postpartum mice and normal mice. (F) Epididymal fat weight. Each datum represents the mean \pm S.E.M. of three independent experiments. ${ }^{\#} p<0.05$; significantly different from the control (10\% kcal\% fat diet) value. ${ }^{*} p<0.05$; significantly different from the HF (60\% kcal\% fat diet) value. 


\section{RESULTS}

\section{Effects of GGT on HF diet-induced increase of weight}

The initial body weights of the mice, prior to feeding with CON or HF diet, did not significantly differ (Fig. 1A); however, there was a significant increase of body weight in the HF group compared with the CON group after 6 weeks $(p<0.05$, Fig. 1A). The average daily food intake of the HF group was significantly less than that of the CON group (Fig. 1C). On the other hand, weight increases in the GGT group were significantly less than those of the HF group ( $p<0.05$, Fig. 1A). At the end of the experimental period, the HF group gained $4.30 \pm 0.84 \mathrm{~g}$ of weight, whereas the GGT group gained only $0.96 \pm 0.35 \mathrm{~g}$ (Fig. 1A). The feed gain ratio was calculated by dividing the weight increase by food intake. As shown in Fig. $1 \mathrm{D}$, the feed gain ratio of the HF group was significantly increased, whereas the GGT group had a significantly decreased feed gain ratio $(p<0.05)$. In terms of food intake, a postpartum mouse has a tendency to eat more than normal (virgin) mouse (Fig. 1E). In addition, the weights of epididymal fat tissues in the HF group significantly increased more than those of the CON group $(p<0.05$, Fig. 1F). However, the oral administration of GGT significantly inhibited the increase of epididymal fat tissues $(p<0.05$, Fig. $1 \mathrm{~F})$.

\section{Effects of GGT on HF diet-induced increase of plasma lipid profiles}

$\mathrm{HF}$ feeding resulted in a significant increase in the plasma triglyceride, LDL-cholesterol, total cholesterol, and GPT levels in the mice ( $p<0.05$, Fig. 2). However, the oral administration of GGT inhibited the increase of plasma triglyceride in the mice fed HF diet ( $p<0.05$, Fig. 2A). In addition, GGT inhibited the increases of LDL-cholesterol and total cholesterol in the serum of mice fed HF diet ( $p<0.05$, Fig. 2B and C). Finally, the oral administration of GGT inhibited the increase of plasma GPT levels in the mice fed HF diet ( $p<0.05$, Fig. 2D). The inhibition rates of GGT on the plasma triglyceride, LDL-cholesterol, total cholesterol, and GPT levels were 77.94 $\pm 2.55,69.23 \pm 22.21,73.91 \pm 8.37$, and $39.29 \pm 10.31$, respectively.
A
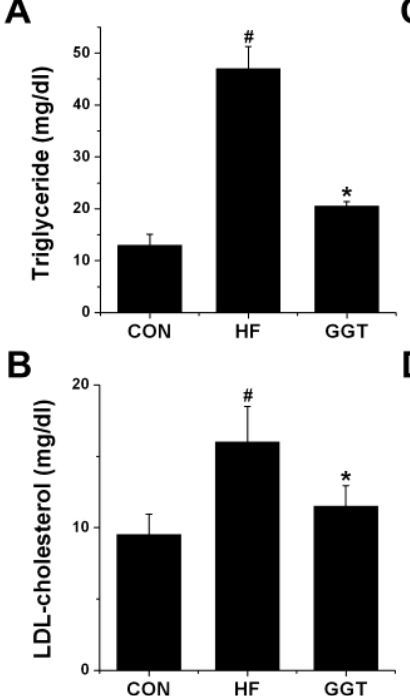

C

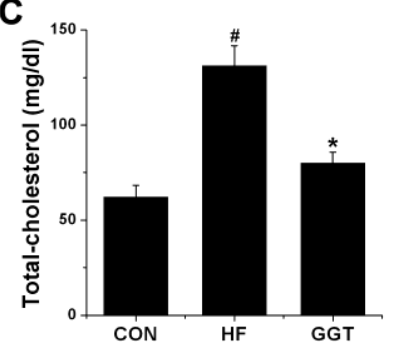

D

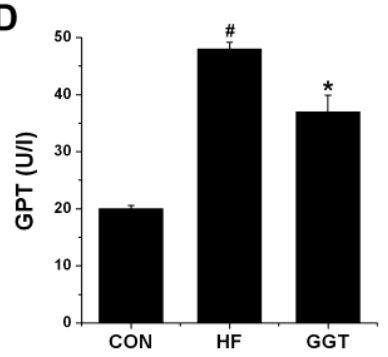

Fig. 2. Effects of GGT on HF diet-induced increase of plasma lipid profiles. Plasma concentrations of (A) triglyceride (B) LDL-cholesterol (C) total-cholesterol (D) GPT. Each datum represents the mean \pm S.E.M. of three independent experiments. ${ }^{\#} p<0.05$; significantly different from the control $(10 \% \mathrm{kcal} \%$ fat diet $)$ value. ${ }^{*} p<0.05$; significantly different from the HF $(60 \% \mathrm{kcal} \%$ fat diet $)$ value.

TANG / www.e-tang.org

\section{DISCUSSION}

Regarding socioeconomic factors, researches are consistently reported that the level of socioeconomic status is inversely correlated with obesity in developed countries (Gustafsson et al., 2012). Low income women with less than a high school education have twice the obesity rates as women of higher socioeconomic status (Singh et al., 2010). Postpartum women having limited financial and social resources are potential risk groups for weight control and chronically high stress levels in later life. In this study, we show that GGT inhibit not only the increases of body weight and adipose tissues in the postpartum mice fed HF diet, but also the increases of plasma lipid profiles such as triglyceride, LDL-cholesterol, total cholesterol, and GPT. GGT is well-known as a Korean Medicine for the removal of static blood. These results indicate that GGT could help the increase of body weight after childbirth, especially in the lower income bracket.

The obesity epidemic, including a marked increase in the prevalence of obesity among pregnant women, represents a critical public health problem throughout the world (Lau et al., 2011). This maternal obesity in pregnancy is associated with a number of adverse outcomes for mother and her offspring both perinatally and later in life (Van Lieshout et al., 2011). Lee et al. (2010) reported that on average, body weight increased $26.54 \%$ $(13.95 \mathrm{~kg})$ from pre-pregnancy to 36 - 39 weeks of pregnancy and body weight remained $6.26 \%(3.29 \mathrm{~kg})$ higher at $24-25$ weeks postpartum compared with pre-pregnancy. In traditional Korean medical clinics, many clinical doctors prescribe GGT for mother to get rid of lochia. With the removal of the lochia, most mothers would recover their health. However, if the lochia was not removed after childbirth, many postpartum disorders, such as postpartum obesity and postpartum depression, would occur. Therefore, we hypothesize that static blood is the main cause of postpartum obesity.

It has been reported that $\mathrm{HF}$ diet induces significant increase of body weight ( $\mathrm{Ng}$ et al., 2010). Our results also showed that HF diet induced a significant increase of body weight in postpartum mice (Fig. 1A). Even though the average daily food intake of the HF group was less than that of the CON group, the increase of body weight in the HF group was larger than that of the CON group, showing the efficacy of the $\mathrm{HF}$ diet. In our results, GGT significantly inhibited the increase of body weight and feed gain ratio; thus we can presume that GGT would be effective in the recovery of body weight for a mother.

It has been reported that the intake of mothers was more than normal (Whichelow, 1975). Bermudez et al. (1989) also reported that daily intake was increased after parturition in sheep. Although it is not significant, our results also showed that the food intake of the postpartum mouse was larger than that of the normal mouse (Fig. 1E). Thus, we can assume that eating habits would continue after parturition.

$\mathrm{Ng}$ et al. (2010) also reported that a HF diet induces a significant increase in fat content. Consistent with the previous report, our results also showed that a $\mathrm{HF}$ diet induced a significant increase in epididymal fat tissues (Fig. 1F). The oral administration of GGT inhibited this increase and this result indicates that GGT has a weight-lowering effect especially in the weight of fat tissues.

Obesity is described as an excess amount of body fat and is also associated with several risk factors of lipid metabolic syndrome such as elevated triglyceride concentrations and high LDL-cholesterol levels (Brown et al., 2009). We showed that GGT inhibited the elevations of triglyceride and LDL-cholesterol as well as total cholesterol in the serum of mice fed a HF diet (Fig. 2A-C). The plasma enzyme GPT is a 
specific marker of liver damage (Ozaki et al., 1995). The reduced activity of this enzyme by GGT indicates decreased hepatic oxidative stress under a HF diet condition. Therefore, we can deduce that GGT would improve the lipid metabolic syndrome through the regulation of plasma lipid profiles without liver damage.

In conclusion, we have shown that GGT can inhibit the increases of body weight and adipose tissues in the postpartum mice fed HF diet. GGT inhibited the elevations of plasma lipid profiles such as triglyceride, LDL-cholesterol, total cholesterol, and GPT. Overall, these results provide proof that GGT can inhibit the postpartum obesity and open new perspective to recover the shape of mother into the moment of conception.

\section{ACKNOWLEDGEMENTS}

This research was supported by Basic Science Research Program through the National Research Foundation of Korea (NRF) funded by the Ministry of Education, Science and Technology(2010-0005591) and by a grant from the Kyung Hee University in 2011 (KHU-20110092).

\section{CONFLICTS OF INTEREST}

The authors have declared no conflict of interest.

\section{REFERENCES}

Bermudez FF, Forbes JM, Jones R. Feed intakes and meal patterns of sheep during pregnancy and lactation, and after weaning. Appetite. 1989;13:211-222.

Brown WV, Fujioka K, Wilson PW, Woodworth KA. Obesity: why be concerned?. Am J Med. 2009;122:S4-S11.

Carter AS, Wood Baker C, Brownell KD. Body mass index, eating attitudes, and symptoms of depression and anxiety in pregnancy and the postpartum period. Psychosom Med. 2000;67:264-270.

Choi BH. Effect of Cnidii Rhizoma Water Extract on the Cerebral Blood Flow. (Iksan, Republic of Korea: Master's thesis from Wonkwang University), 2004.

Davis EM, Stange KC, Horwitz RI. Childbearing, stress and obesity disparities in women: a public health perspective. Matern Child Health J. 2012;16:109-118.

Friedewald WT, Levy RI, Fredrickson DS. Estimation of the concentration of low-density lipoprotein cholesterol in plasma, without use of the preparative ultracentrifuge. Clin Chem. 1972;18:499-502.

Gustafsson PE, Persson M, Hammarstrom A. Socio-economic disadvantage and body mass over the life course in women and men: results from the Northern Swedish Cohort. Eur J Public Health. 2012;22:322-327.

Hong WS. HwangJeNaeGyeongYeongChu. (Seoul, Republic of Korea: Oriental Medicine Research Center Press), 1985.
Huh J. DongUiBoGam. (Seoul, Republic of Korea: BUBIN PUBLISHERS CO.), 1999.

Kim DI, Kim SW, Kim JH, Kim CW, Kim HJ, Baek SH, Song BK, Yu DY, Yu SG, Yun MH, Lee KS, Lee DG, Lee DN, Lee IS, Lee TK, Lee HJ, Im EM, Jang JB, Jeong JH, Jo JH, Jo HB, Jin CS. HanBangYeoSeongUiHak (Seoul, Republic of Korea: JEONGDAM BOOKS), 2007.

Kim WH, Choi DY. JangBuByeonJeungRonChi (Seoul, Republic of Korea: SEONGBOSA), 1985.

Lau C, Rogers JM, Desai M, Ross MG. Fetal programming of adult disease: implications for prenatal care. Obstet Gynecol. 2011;117:978-985.

Lee CF, Hwang FM, Liou YM, Chien LY. A preliminary study on the pattern of weight change from pregnancy to 6 months postpartum: a latent growth model approach. Int J Obes (Lond). 2011;35:1079-1086.

Mokdad AH, Bowman BA, Ford ES, Vinicor F, Marks JS, Koplan JP. The continuing epidemics of obesity and diabetes in the United States. JAMA. 2001;286:1195-1200.

Ng SF, Lin RC, Laybutt DR, Barres R, Owens JA, Morris MJ. Chronic high-fat diet in fathers programs $\beta$-cell dysfunction in female rat offspring. Nature. 2010;467:963-966.

Ozaki M, Fuchinoue S, Teraoka S, Ota K. The in vivo cytoprotection of ascorbic acid against ischemia/reoxygenation injury of rat liver. Arch Biochem Biophys. 1995;318:439-445.

Pedersen P, Baker JL, Henriksen, TB, Lissner L, Heitmann BL, Sorensen T, Nohr EA. Influence of psychosocial factors on postpartum weight retention. Obesity. 2011;19:639-646.

Pitt B, Mancini GB, Ellis SG, Rosman HS, Park JS, McGovern ME. Pravastatin limitation of atherosclerosis in the coronary arteries (PLAC I): reduction in atherosclerosis progression and clinical events. PLAC I investigation. J Am Coll Cardiol. 1995;26:1133-1139.

Rasouli N, Kern PA. Adipocytokines and the Metabolic Complications of Obesity. J Clin Endocrinol Metab. 2008;93:S64-S73.

Reck C, Hunt A, Fuchs T, Weiss R, Noon A, Moehler E, Downing G, Tronick EZ, Mundt C. Interactive regulation of affect in postpartum depressed mothers and their infants: an overview. Psychopathology. 2004;37:272-280.

Schmitt NM, Nicholson WK, Schmitt J. The association of pregnancy and the development of obesity: results of a systematic review and meta-analysis on the natural history of postpartum weight retention. Int $\mathrm{J}$ Obes (Lond). 2007;31:1642-1651.

Singh GK, Siahpush M, Hiatt RA, Timsina LR. Dramatic increases in obesity and overweight prevalence and body mass index among ethnic-immigrant and social class groups in the United States: 1976-2008. J Community Health. 2011;36:94-110.

Stienstra R, Duval C, Müller M, Kersten S. PPARs, Obesity, 
and Inflammation. PPAR Res. 2007;2007:95974.

Sundaram S, Harman JS, Peoples-Sheps MD, Hall AG, Simpson SH. Obesity and Postpartum Depression: Does Prenatal Care Utilization Make a Difference?. Matern Child Health J. 2012;16:656-667.
Van Lieshout RJ, Taylor VH, Boyle MH. Pre-pregnancy and pregnancy obesity and neurodevelopmental outcomes in offspring: a systematic review. Obes Rev. 2011;12:e548-e559.

Whichelow MJ. Letter: Calorie requirements for successful breast feeding. Arch Dis Child. 1975;50:669. 